\title{
Metacommunity patterns in larval odonates
}

\author{
Shannon J. McCauley • Christopher J. Davis · \\ Rick A. Relyea $\cdot$ Kerry L. Yurewicz $\cdot$ \\ David K. Skelly · Earl E. Werner
}

\begin{abstract}
The growth of metacommunity ecology as a subdiscipline has increased interest in how processes at different spatial scales structure communities. However, there is still a significant knowledge gap with respect to relating the action of niche- and dispersal-assembly mechanisms to observed species distributions across gradients. Surveys of the larval dragonfly community (Odonata: Anisoptera) in 57 lakes and ponds in southeast Michigan were used to evaluate hypotheses about the processes regulating community structure in this system. We considered the roles of both niche- and dispersal-assembly processes in
\end{abstract}

S. J. McCauley · C. J. Davis · E. E. Werner

Department of Ecology and Evolutionary Biology,

University of Michigan, Ann Arbor, MI 48109-1048, USA

\section{R. A. Relyea}

Department of Biological Sciences, University of Pittsburgh, Pittsburgh, PA 15260, USA

\section{K. L. Yurewicz}

Biological Sciences, Plymouth State University,

Plymouth, NH 03264, USA

\section{K. Skelly}

Department of Ecology and Evolutionary Biology, School of Forestry and Environmental Studies,

Yale University, New Haven, CT 06511, USA

\section{S. J. McCauley (}

Center for Population Biology, University of California, 2320 Storer Hall, One Shields Ave., Davis, CA 95616, USA determining patterns of species richness and composition across a habitat gradient involving changes in the extent of habitat permanence, canopy cover, area, and top predator type. We compared observed richness patterns and species distributions in this system to patterns predicted by four general community models: species sorting related to adaptive trade-offs, a developmental constraints hypothesis, dispersal assembly, and a neutral community assemblage. Our results supported neither the developmental constraints nor the neutral-assemblage models. Observed patterns of richness and species distributions were consistent with patterns expected when adaptive tradeoffs and dispersal-assembly mechanisms affect community structure. Adaptive tradeoffs appeared to be important in limiting the distributions of species which segregate across the habitat gradient. However, dispersal was important in shaping the distributions of species that utilize habitats with a broad range of hydroperiods and alternative top predator types. Our results also suggest that the relative importance of these mechanisms may change across this habitat gradient and that a metacommunity perspective which incorporates both niche- and dispersal-assembly processes is necessary to understand how communities are organized.

Keywords Local-regional - Metacommunity . Habitat gradient $\cdot$ Niche assembly $\cdot$ Dispersal assembly

\section{Introduction}

Despite sharing a common regional species pool, local environments typically show substantial variation in both richness and composition. Ecologists have long been interested in the processes that structure this variation. Nevertheless, our understanding of these processes remains 
fragmentary. Two disparate perspectives on this issue, dispersal assembly and niche assembly, have recently been combined in metacommunity models predicting the compositions of local communities (Cornell and Lawton 1992; Holyoak et al. 2005; Leibold et al. 2004). Comparative and experimental studies assessing the relative contributions of niche- and dispersal-assembly processes have typically found that both sets of processes impact community structure (Chase 2003; Cottenie et al. 2001; Cottenie and De Meester 2004; Friedenberg 2003; Kneitel and Miller 2003; Shurin 2001; Tilman 1997; Tofts and Silvertown 2002; Wright et al. 2003). However, these empirical studies have rarely been conducted in the context of the patterns of species distributions across natural habitat gradients, despite the potential for this approach to provide useful tests of metacommunity models (but see Urban 2004). This is a critical gap because the relative importance of niche- and dispersal-assembly processes may change across habitat gradients as different factors become limiting, and thus studies of such gradients can indicate what factors influence the relative importance of different community processes.

We examined the distributional patterns in a group of freshwater invertebrates, larval dragonflies (Odonata: Anisoptera), across a broad habitat gradient of lentic systems ranging from temporary ponds to permanent lakes. We examined the fit between the observed habitat-community patterns and four models of community organization to assess their relative contributions to community structure in this system; two niche-assembly mechanisms (adaptive trade-offs or developmental constraints), one dispersalassembly mechanism (including mass effects and sourcesink dynamics), and finally a neutral model of communities in which species are functionally equivalent (Hubbell 2001) (Table 1). By directly comparing observed patterns of community structure with patterns predicted by general models, we gain insight into the processes that act to structure these communities and how factors that are critical in limiting community composition can shift across habitat gradients.

\section{Background and model predictions}

Model predictions are summarized in Table 1. It should be noted that some predictions apply to multiple models. For example, predictions related to species sorting across the habitat gradient are applicable when testing both nicheassembly and neutral models. Additionally, these models are not necessarily mutually exclusive and multiple mechanisms are likely to act in this system. Rather than assuming that patterns observed at this spatial and temporal scale will provide support for a single model, we combined multiple lines of evidence to assess how these processes may contribute to the observed community assemblage in these ponds.
Adaptive trade-offs can structure species distributions across habitat gradients. In freshwater lentic systems, increased permanence (longer hydroperiod) is associated with increased abundance and diversity of predators, and changes in top predator type ranging from large invertebrate predators to fish (Wellborn et al. 1996). The relationship between top predator type and habitat permanence can result in species-sorting across this gradient because of the presence of fundamental trade-offs (Wellborn et al. 1996). The rapid life-cycles required to utilize less permanent habitats are often associated with high levels of activity, which make species more vulnerable to predators (Johansson and Suhling 2004; Skelly 1995; Wellborn et al. 1996; Werner and Anholt 1993). Consequently, differential resolution of this developmental rate/predation risk trade-off by species would lead to the segregation of species across the hydroperiod-predator type gradient, with distinct turnover in suites of species associated with the transition in predator community. Additionally, this model predicts that species with different development rates will be nonrandomly distributed across the top predator transition, with slower developing species found in more permanent habitats with high-risk predators, and rapidly developing species being less common or excluded from these habitats.

If species are not restricted by adaptive trade-offs to habitats at either end of the permanence-predator intensity gradient, but are instead successively filtered from the community by constraints of developmental period in relation to habitat permanence, we would expect to find nested communities (Patterson and Atmar 1986). This developmental constraints hypothesis predicts that species richness will decline with decreasing permanence, and that species will occur across the full range of habitat permanence conditions in which they can complete development (Schneider and Frost 1996).

Dispersal-assembly processes can also interact with habitat permanence to affect the richness and composition of communities. Species with low dispersal and colonization rates may be absent from otherwise suitable habitats that experience periodic disturbance through drying. Similarly, species with high dispersal and colonization rates may be present in sites where they cannot maintain populations (e.g., mass effects, Shmida and Wilson 1985) or in sites where they contribute to the regional population in some but not all periods (e.g., weak source-sink dynamics, Holt 1997). If dispersal is important in determining community structure, we would predict that species will regularly colonize habitats which are at least intermittent sinks, resulting in a breakdown between species life-history requirements and their distributions. Dispersal thus may have an important role in maintaining diversity both locally and regionally (Vandvik and Goldberg 2006), and we examine the effects of dispersal on both aspects of diversity. 


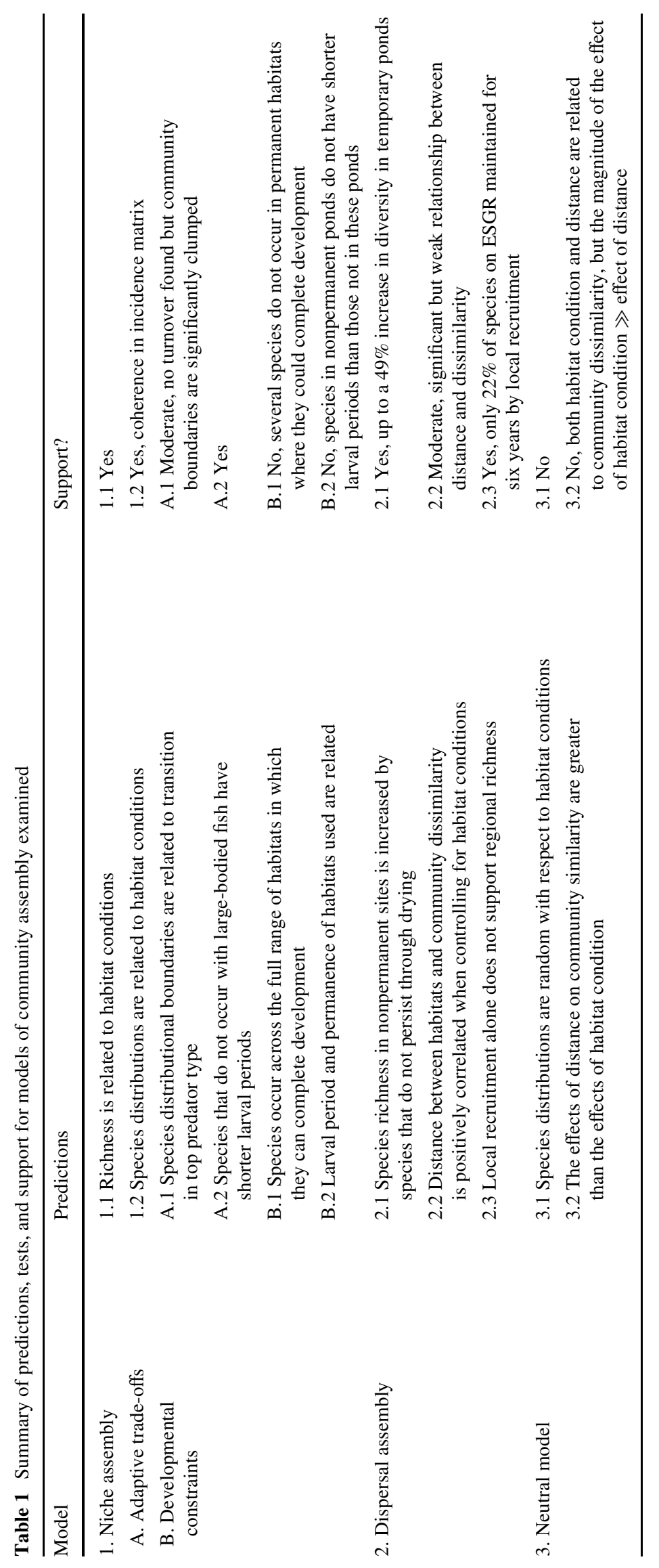


The neutral community perspective (Hubbell 2001) provides an alternative null model to the three models above which assumes that species respond differently to aspects of the habitat gradient (Bell 2001). The neutral model assumes that species are functionally equivalent and that species richness within a habitat is set by habitat characteristics that determine the probability of species loss and gain in local sites. Critically, the neutral model predicts that dispersal limitation will have a greater impact on community assembly than species-sorting and adaptation to local conditions (Gilbert and Lechowicz 2004).

\section{Methods}

\section{Study system}

We combined the results of two surveys of larval dragonflies, conducted with similar methodologies, to examine patterns of species richness and distributions across a broader environmental gradient than either survey alone encompassed. This resulted in a combined dataset from 57 lakes and ponds. The water bodies surveyed encompassed the majority of lentic habitat types in the region, from small, shaded, ephemeral ponds in which invertebrates were the dominant predators to large, open-canopy, permanent lakes with large-bodied predatory fish.

One survey was conducted on the Edwin S. George Reserve (hereafter: ESGR). The ESGR is a research facility near Pinckney, Michigan $\left(42^{\circ} 28^{\prime} \mathrm{N}, 84^{\circ} 00^{\prime} \mathrm{W}\right)$, administered by the Department of Ecology and Evolutionary Biology at the University of Michigan (site description: Skelly et al. 1999). The results presented here encompass six years of surveying 35 ponds twice yearly (in May and July) on the ESGR from 1996 to 2001.

The second survey sampled water bodies in three areas within the same region as the ESGR (all sites were located within $40 \mathrm{~km}$ of the ESGR) located in terrestrial environments similar to the ESGR. These three sites were the Pinckney Recreation Area, which surrounds the ESGR, the Waterloo State Recreation Area (24 km west of the ESGR, $42^{\circ} 21^{\prime} \mathrm{N}, 84^{\circ} 04^{\prime} \mathrm{W}$ ), and one city park located in Ann Arbor, MI $\left(33 \mathrm{~km}\right.$ southeast of the ESGR, $42^{\circ} 17^{\prime} \mathrm{N}$, $83^{\circ} 44^{\prime} \mathrm{W}$ ). Water bodies surveyed included 22 lakes and ponds that spanned the full habitat gradient examined. Results presented here are from surveys conducted four times per year (once monthly May-August) in 2000 and 2001. Although this protocol resulted in more sampling periods across the year than in the ESGR survey, the larval phenology of species in these lakes and ponds meant that their detection should be equally likely under both sampling regimes. To assess this, we compared the relationship between richness and habitat characteristics (described below) with and without these additional sampling periods ("Results"). To increase the sample size of large lakes with large-bodied fish, three lakes of this type were added to the survey in 2001. These additional samples did not change richness estimates as there was no difference in the cumulative richness of the lakes with large-bodied fish sampled in 2000 and 2001 and those sampled only in 2001 (independent samples $t$ test: $t=1.6, d f=8, P=0.139$ ).

A total of 43 species of larval dragonfly were collected from these 57 water bodies. These species represent four families of anisopteran odonates: Aeshnidae (nine species), Corduliidae (six species), Gomphidae (three species), and Libellulidae (25 species). Comparisons of these results to regional species lists (O'Brien 2008) suggest that our surveys encompassed the majority of species found in lentic water bodies in the area. The majority ( $\sim 94 \%$ in both surveys) of specimens collected were identified to species. Identifications were conducted by two researchers who used consistent criteria to identify specimens.

\section{Environmental gradients}

Habitat area was measured for all sites on the ESGR using aerial photographs taken in the winter of 1995 (Werner et al. 2007). For sites off the ESGR, measurements of habitat area for large, named lakes were available in public records at the Institute for Fisheries Research, Michigan DNR. Other lakes were measured using aerial photographs taken by the Michigan DNR. We scanned these into Adobe Photoshop $^{\odot}$ and used pixel counts to estimate lake areas based on counts for lakes of known area in the same photos. Finally, small habitats that could not be identified on the aerial photos were measured in the field. Area was natural$\log$-transformed $\left(\ln \left(\right.\right.$ area in $\left.\left.\mathrm{m}^{2}\right)\right)$ for analyses.

Canopy cover was measured with a spherical densiometer using methods developed by Skelly et al. (2006). Canopy cover was measured for all habitats on the ESGR and for eight sites off the ESGR. Canopy was not measured at large $\left(>5,000 \mathrm{~m}^{2}\right)$, permanent lakes where trees are confined to shore margins and cannot penetrate significant portions of the lake area, and at one mid-sized pond located in a grass and wetland complex with no trees adjacent to the site. These sites were all estimated to have $0 \%$ canopy cover.

Hydroperiod, the length of time each site held water, was recorded for sites located on the ESGR from 1996 onwards, while hydroperiod was recorded for all sites in 2000-2002. Details of measurements of hydroperiod in ponds on the ESGR are provided by Werner et al. (2007). Hydroperiod for sites off the ESGR was measured as the presence or absence of water during the four survey periods, the third week of each month from May to August. Sites that held water but had lowered water levels in August were monitored 
through October to assess whether any drying occurred. Because of cool temperatures and associated low evaporation rates after this period, ponds which hold water through October rarely dry further after this time. For both surveys, if a pond held water in one survey but was dry in the next survey it was estimated to have dried at the midway point between observations. For some analyses we characterized water bodies as either permanent or nonpermanent, rather than using a continuous variable. Permanent sites were those which held water through all the years we surveyed them, while nonpermanent sites were those which dried at least once during the years in which they were surveyed. Therefore, nonpermanent sites include ponds that can be considered temporary (they dry in most years), as well as semipermanent ponds that can hold water for multiple years but dry in some conditions.

Sites were also characterized on the basis of top predator type. Water bodies on the ESGR include habitats with no fish and habitats that had small-bodied fish (either continually or intermittently over the survey period), principally the eastern mudminnows (Umbra limi) and the redside dace (Phoxinus eos). Dipnet sampling and seine nets were used to sample fish communities on the ESGR (details in Werner et al. 2007). Sites were characterized for analyses as invertebrate sites (never had fish) or small-bodied fish sites (had one or more of these fish species either continually or intermittently).

In sites off the ESGR, permanent lakes and ponds $>1 \mathrm{~m}$ deep were all seined in May and June of 2001 to determine the composition of the predator community. In spring 2001, minnow trapping was conducted in water bodies $<1 \mathrm{~m}$ deep, and these ponds were dipnetted during all survey periods. Comparisons of results from dipnetting and minnow trapping from the same time period indicate that the two methods had equivalent probabilities of detecting fish presences. In sites without fish, invertebrates were the top predators. Sites with small-bodied predatory fish were dominated by the eastern mudminnow ( $U$. limi). Sites with large-bodied predatory fish had diverse fish communities, including multiple small-bodied fish species, but bluegill (Lepomis macrochirus) and pumpkinseed (Lepomis gibbosus) sunfish were the most common large-bodied fish. Based on the combination of sampling methods, water bodies were characterized for analyses as invertebrate sites, small-bodied fish sites, or large-bodied fish sites.

\section{Dragonfly surveys}

Sites on the ESGR were sampled twice per year from 1996 through 2001 as part of a broader taxonomic survey of amphibians and their predators in these water bodies. Sampling was conducted with pipe samples (Skelly 1996) and dipnetting (protocols: Werner et al. 2007).
Sites off the ESGR were surveyed using D-framed dipnets swept through ca. $0.5 \mathrm{~m}$ of vegetation or benthic sediments and emptied into sorting trays. Dragonfly larvae were obtained by sorting through the vegetation and sediments in the trays and preserving larvae in $70 \%$ ethanol for later identification. Dipnetting in each water body was conducted for a predetermined number of person-minutes. The length of this sampling period was determined by habitat size, adjusted for ease of movement through habitats. The minimum sampling effort was 60 person-min of dipnetting conducted in $<1 \mathrm{~m}$ depth in all habitats, which allowed us to sample two approximately $2 \times 100 \mathrm{~m}$ transects of littoral habitat. All small ponds $\left(<1,900 \mathrm{~m}^{2}\right.$ and $<1 \mathrm{~m}$ deep $)$ were sampled with this effort. This same protocol was applied to the littoral regions $(<1 \mathrm{~m}$ deep) of larger water bodies, but the sampling effort was additionally increased to cover the broader range of habitat depths available in larger sites. Standard sampling periods were: 90 person-min in midsized lakes (between 29,000 and $1,900 \mathrm{~m}^{2}$ ) and 120 personmin in large lakes $\left(\geq 30,000 \mathrm{~m}^{2}\right)$. In one mid-sized lake we increased this to 120 person-min because the difficulty of movement slowed the sampling rate. In the large lakes, 40 person-min out of the 120 person-min sampling period was conducted from a kayak to access areas too deep to reach on foot $(\sim 2 \mathrm{~m})$. Because of maneuverability issues, one site (Green Lake) was sampled using a kayak for the entire 120 person-min period. As in other lakes, the littoral area was sampled for 80 person-min from a kayak before sampling in deeper water for the remaining 40 person-min. To ensure adequate sampling, we also adjusted the above times as follows. If a new genus was found in the last $10 \mathrm{~min}$ of the predetermined sampling period, sampling was continued for an additional $10 \mathrm{~min}$, and this was repeated until $10 \mathrm{~min}$ had passed without a new genus being collected.

In both surveys, sampling effort was adjusted based on pond size, and a critical assumption of our protocols was that these adjustments resulted in equivalent detection probabilities for species across sites. To assess this assumption, we used species accumulation curves based on resampling and an analysis of the fraction of singletons (species represented by a single individual) in habitats of different sizes. The presence of many singletons in samples from a site can suggest that richness is underestimated (Colwell and Coddington 1994), and differences between sites in the fraction of species represented by singletons would suggest that we were differentially estimating the true richness in these habitats. We examined species accumulation curves for water bodies of each size class using EcoSim (Gotelli and Entsminger 2001). Species accumulation curves for habitats of the three size classes were constructed by pooling individuals of a given species from all ponds in each size class, and then these were randomly resampled by EcoSim across a range of abundances up to the total number of individuals sampled from habitats of a given size, 
and the diversity for each resampled abundance level was calculated. We plotted the increase in diversity across a range of resampled abundances for the three size classes of pond, and all of these curves reached an asymptote at which diversity remained essentially constant with continued resampling. That the curves had reached an asymptote was determined based on a $0 \%$ increase in median diversity and a $\leq 3 \%$ increase in mean species diversity with continued resampling after reaching $80 \%$ of the total abundance from the three pond types. These results suggest that we adequately sampled water bodies to estimate the true richness of dragonfly communities in these sites. However, to further assess whether species detection probabilities were similar across water bodies of different sizes we compared the fraction of species represented by singletons (number singletons/number of species) in each pond and compared whether ponds from the three size categories differed in the proportion of species represented by singletons (Colwell and Coddington 1994). We found no difference in the fraction of species represented by singletons in the ponds in the three size categories $\left(F_{(2,54)}=0.079\right.$, $P=0.924)$. The combination of results from the species accumulation curves and the fraction of singletons in differentsized water bodies suggest that species detection probabilities did not markedly change across the gradient of water-body sizes.

Presence-absence data from the ESGR and PinckneyWaterloo surveys were combined in analyses. Three lines of evidence indicate that the presence-absence data from these surveys were comparable and suitable to combine. First, species found in both surveys had the same distributions across environmental gradients, and the species composition of sites grouped by their habitat characteristics was highly similar between the two surveys. Specifically, while the non-ESGR sites contained a unique habitat type, lakes with large-bodied fish, the non-ESGR survey contained ponds across the full habitat gradient (see the "Electronic Supplementary Material"). We found a unique set of species in the survey done off the ESGR, but these species were only found in those lakes with large-bodied fish and not in other non-ESGR sites, although the same methods were used in the entire survey. This provides good evidence that we found these species in the non-ESGR survey because of the unique habitat type included and not because of the sampling methodology. Second, the species detected only in lakes with large-bodied fish were not detected on the ESGR despite extensive dipnetting using the same techniques as the off-ESGR survey (McCauley and Davis, personal observation). Finally, there was no difference in the residuals from the two surveys in the analysis relating cumulative richness to the PC scores of habitat conditions (independent $t$ test: $t=0.066, d f=55, P=0.947$ ). Therefore, richness estimates for ponds with similar habitat characteristics did not differ between the two surveys.
To assess the distribution of life-history traits across habitat conditions, we compiled these traits for species collected in the survey from the literature (Walker and Corbet 1978) and from our own data. Larval developmental time ranged from six months for univoltine species that overwinter as eggs and subsequently complete larval development, to two years for semivoltine species. The estimate of larval developmental time was related to habitat distribution using coded variables. For each habitat distribution variable (hydroperiod, permanent or nonpermanent, and top predator type, invertebrate, small-bodied fish, large-bodied fish), species were scored as either 1 or 0 to indicate their presence or absence in sites with these characteristics.

\section{Data analysis}

The variables used to describe habitat conditions (hydroperiod, canopy cover, and area) are fundamentally linked through biological processes (e.g., the relationships between area and canopy cover are a function of the tolerance of trees to inundation) and are strongly correlated with each other. Therefore, we used PCA to derive a descriptor of the habitat conditions based on these three habitat variables characterizing each water body. Principal components were retained using broken-stick criteria (Jackson 1993). The relationship between site PC scores and cumulative species richness was assessed using linear regression. The effect of top predator type was examined using an ANCOVA with top predator type (invertebrate, small bodied fish, or large-bodied fish) as a fixed factor and PC scores as a covariate. Post hoc comparisons were made using Bonferroni contrasts. All analyses were conducted in SPSS 11.5.

Species distributions were summarized using the incidence matrix, a matrix of species presence/absence data by site (Fig. 2; see "Electronic Supplementary Material"). We used the approach and software developed by Leibold and Mikkelson (2002), which provides a synthetic analysis of the incidence matrix, testing for patterns of nestedness or turnover in a single analysis. Although other methods exist to test for either of these patterns (e.g., nestedness: Patterson and Atmar 1986), this method is unique in testing for multiple patterns in a single analysis. This approach ordinates sites and species (column and row in the matrix, respectively) by reciprocal averaging, which arranges the incidence matrix to place sites with the most similar species compositions and species with the most similar distributions close together. The observed matrix is then compared to randomized rearrangements of the matrix to test for coherence.

Coherence is defined as a strong dominant axis of variation, the degree to which the pattern of species presences across sites can be collapsed into a single dimension. In a 
completely coherent matrix, ordinated species presences in sites would not be interrupted by species absences. If the ordinated matrix is not coherent, the arrangement of species and sites by reciprocal averaging will produce as many gaps in species distributions as the randomly arranged matrix. If there is coherence, further matrix randomizations are performed to test for nesting or turnover in species and communities and for species and community boundary clumping. Communities are defined as suites of species which have similar distributions within the ordinated matrix. Turnover is the replacement of species or suites of species along the dominant axis of variation in a coherent matrix. Nestedness involves the accumulation of species along the dominant axis such that as richness increases species are added and not replaced. Boundary clumping refers to how grouped (clumped) the edges of individual species distributions, or the distributions of species groups, are across a coherent matrix. Each of these tests is performed by comparing the observed matrix to a minimum of 200 iterations of the randomized matrix. Species found in only one water body were dropped prior to analysis because it is not possible to interpret their habitat distributions based on a single site.

The incidence matrix used in the analysis included 57 sites and 38 species. Species presence was based on cumulative presence, i.e., species were counted as present in a site if they occurred there once at any point in the sampling period. This is a liberal estimate of species distributions, but given the high level of flux in these communities we wanted to investigate where species could potentially occur, since absence may be a function of population cycles or other factors unrelated to habitat characteristics. We used a test for coherence that requires row and column totals for the randomized matrix to match those of the observed matrix. This is a conservative approach to the randomization and limits the probability of type I errors (finding coherence when it does not exist) (Leibold and Mikkelson 2002).

To test our predictions about the relationship between developmental rate and the use of habitats characterized by permanence and predators, we used two $t$ tests to compare the length of the larval developmental period in species that differ in their habitat distributions. One test compared the length of the larval period in species that were found in lakes with large-bodied fish to those that did not occur in these sites. The second $t$ test compared the length of the larval period in species found in non-permanent ponds to those which were not found in nonpermanent ponds.

Relationship between distance, habitat similarity, and community structure

We used partial Mantel tests to examine correlations in distance between habitats, habitat dissimilarity (absolute value of the difference in PC1 scores between sites), and community dissimilarity (equal to: 1 -Jaccard's coefficient of similarity). One partial Mantel test determined the correlation between habitat dissimilarity and community dissimilarity controlling for the effect of distance between habitats. A second partial Mantel examined the correlation between distance and community dissimilarity controlling for habitat dissimilarity. Observed correlations were compared to correlations from the randomized matrix iterated 10,000 times. Analyses were conducted in XLSTAT, Version 2006.3.

Temporal patterns and dispersal

We examined the level of within-site species turnover using the dataset from the ESGR, which has a longer time series for analysis. We calculated the number of extinction and colonization events on the ESGR by assuming that the presence of a species in a site in one year followed by its absence from that site in the next year represents an extinction event, and that the presence of a species after its absence in the previous year is a colonization event. No species in this region overwinters as an adult, so larvae are the appropriate stage to follow for population persistence. We examined the relationship between habitat characteristics and temporal turnover using a partial correlation coefficient. This analysis tested the correlation between pond PC scores and the number of extinction and colonization events in that pond while controlling for cumulative species richness (i.e., removing the variation associated with cumulative richness). We also assessed the role of local recruitment in maintaining regional diversity by determining how many species were found in at least one site on the ESGR in all six years of sampling.

\section{Results}

Environmental variation and species richness

The lakes and ponds surveyed encompassed a broad range of environmental conditions. Sites ranged in area from 7 to $366,244 \mathrm{~m}^{2}$ and in canopy cover from 0 to $90 \%$. We surveyed habitats including ponds that dried every year and sites that never dried (hydroperiod range 0.37-1). There were strong correlations between all three habitat characteristics (Table 2) and each loaded strongly on a single principal component (PC1) that explained $81 \%$ of the measured environmental variation between sites. All three habitat characteristics loaded strongly on this axis: canopy cover $(-0.91)$, hydroperiod $(0.87)$, and area $(0.92)$. No other principal components were retained, as they had eigenvalues of less than 1 (0.2-0.35 vs. 2.4 for the component retained), 
Table 2 Correlations between habitat characteristics measured for the 57 lakes and ponds surveyed

\begin{tabular}{llll}
\hline & Hydroperiod & Canopy cover & $\ln ($ area $)$ \\
\hline Hydroperiod & 1 & -0.68 & $0.69(P<0.001)$ \\
& & $(P<0.001)$ & \\
Canopy cover & 1 & $-0.79(P<0.001)$ \\
$\ln ($ area $)$ & & 1 \\
\hline
\end{tabular}

each explained less than $12 \%$ of the environmental variation, and their retention was not supported by broken-stick criteria (Jackson 1993).

Species richness in water bodies was related to both habitat characteristics and predator type. PC1 scores for water bodies were positively related to cumulative richness in sites $\left(F_{(1,56)}=191.6, P<0.001, R^{2}=0.78\right.$; Fig. 1$)$. We found the same relationship when we dropped results from the additional survey periods (June and August) in the sites not on the ESGR $\left(F_{(1,56)}=182.12, P<0.001, R^{2}=0.77\right)$. Cumulative richness differed significantly across sites with alternative top predator types when PC scores were used as a covariate $\left(F_{(1,53)}=62.6, P<0.001\right.$; predator, $F_{(2,53)}=5.05$, $P=0.01)$. Habitats with large-bodied fish had significantly higher richness than sites with small-bodied fish $(P=0.014)$ and ponds with invertebrate top predators

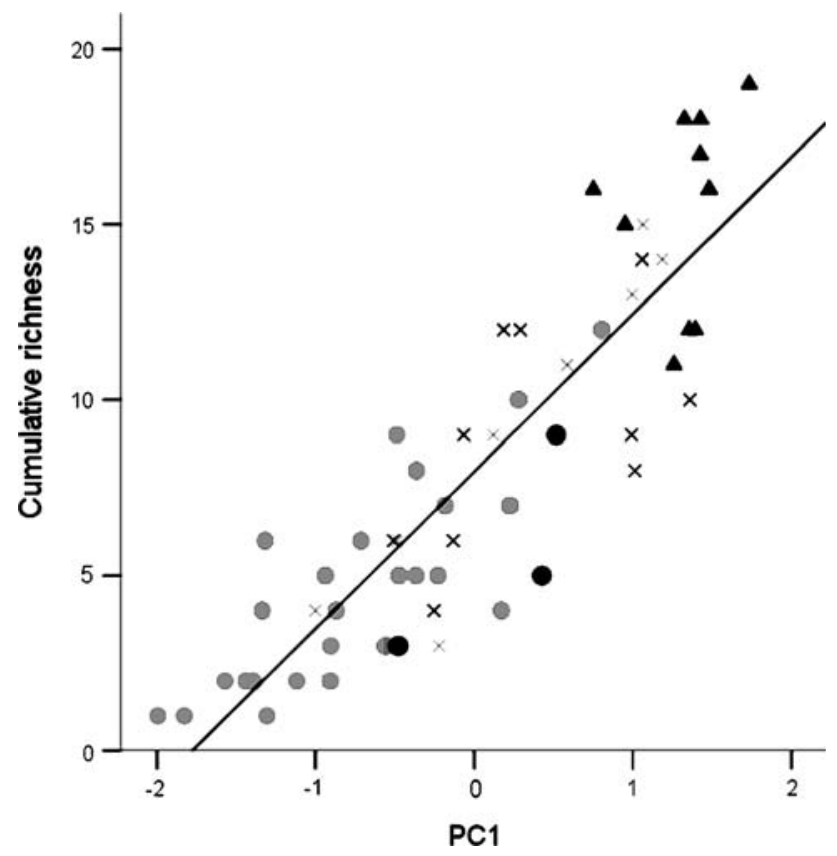

Fig. 1 Cumulative richness of larval dragonflies (Odonata: Anisoptera) in relation to site PC1 scores for 57 sites in southeastern Michigan, USA. Sites include three top predator types: invertebrates (circles), small-bodied fish (crosses), large-bodied fish (triangles). Sites surveyed on the Edwin S. George Reserve (ESGR) are in gray, while sites surveyed off the ESGR are in black
$(P=0.012)$. Ponds with small-bodied fish and invertebrate top predators did not differ in cumulative richness $(P>0.99)$.

Community composition: the incidence matrix

Species were not distributed randomly across sites. The incidence matrix for all sites displayed strong coherence with 529 observed embedded absences in the ordinated matrix, significantly fewer than predicted by the random placement of species in ponds (expected mean \pm 1 sd: $1,076 \pm 61, P<0.001$; Fig. 2). Neither communities nor species displayed significant nestedness or turnover. Species range boundaries were not significantly clumped. Community boundaries were, however, significantly clumped (Morisita's index $=4.65, P<0.0001$ ), indicating that unique suites of species occur in different portions of this gradient. These include nine species that were entirely restricted to permanent habitats with large-bodied fish, while another group of nine species, principally in the genera Aeshna and Sympetrum, never occurred in habitats with large-bodied fish and were principally associated with nonpermanent habitats. However, there were also a number of habitat generalists found across both permanence and top predator transitions, with species accumulating in permanent lakes with large-bodied fish which consequently were the sites of greatest richness (Fig. 2).

Species that did not coexist with large bodied fish had shorter larval periods than species that did coexist with these predators $(t=-2.48, d f=41, P=0.017)$. We found no difference in the larval periods of species that utilized nonpermanent sites and species that were only found in permanent lakes $(t=1.04, d f=41, P=0.305)$.

Relationship between distance, habitat similarity, and community structure

A partial Mantel test found a strong positive correlation between habitat dissimilarity and community dissimilarity while controlling for the effect of distance $(r=0.63$, $P=0.0001)$. Our second partial Mantel test found a significant, but weaker, positive correlation between inter-habitat distance and community dissimilarity while controlling for habitat dissimilarity $(r=0.08, P=0.001)$.

Temporal patterns and dispersal

Richness in nonpermanent sites was increased by species without adaptations to pond drying. In nonpermanent sites, species that require water to overwinter contribute $40 \%$ of the cumulative richness (mean of 2.13 species) in these sites. Additionally, there was no difference in the larval densities of species with and without a diapausing egg stage 
Fig. 2 Species by site incidence matrix ordinated using reciprocal averaging in the ordination program from Leibold and Mikkelson (2002). Species presence is indicated by blackened cells. Species are designated by the first two letters of the genus and first two letters of the species name ("Electronic Supplementary Material,” S1). Sites are numbered and coded by top predator type ( $I$, invertebrates; $S$, small-bodied fish; $L$, large-bodied fish) and by permanence level (gray, permanent sites; unshaded, nonpermanent sites). Site numbers, habitat characteristics, and site location are listed in the "Electronic Supplementary Material;" see S2

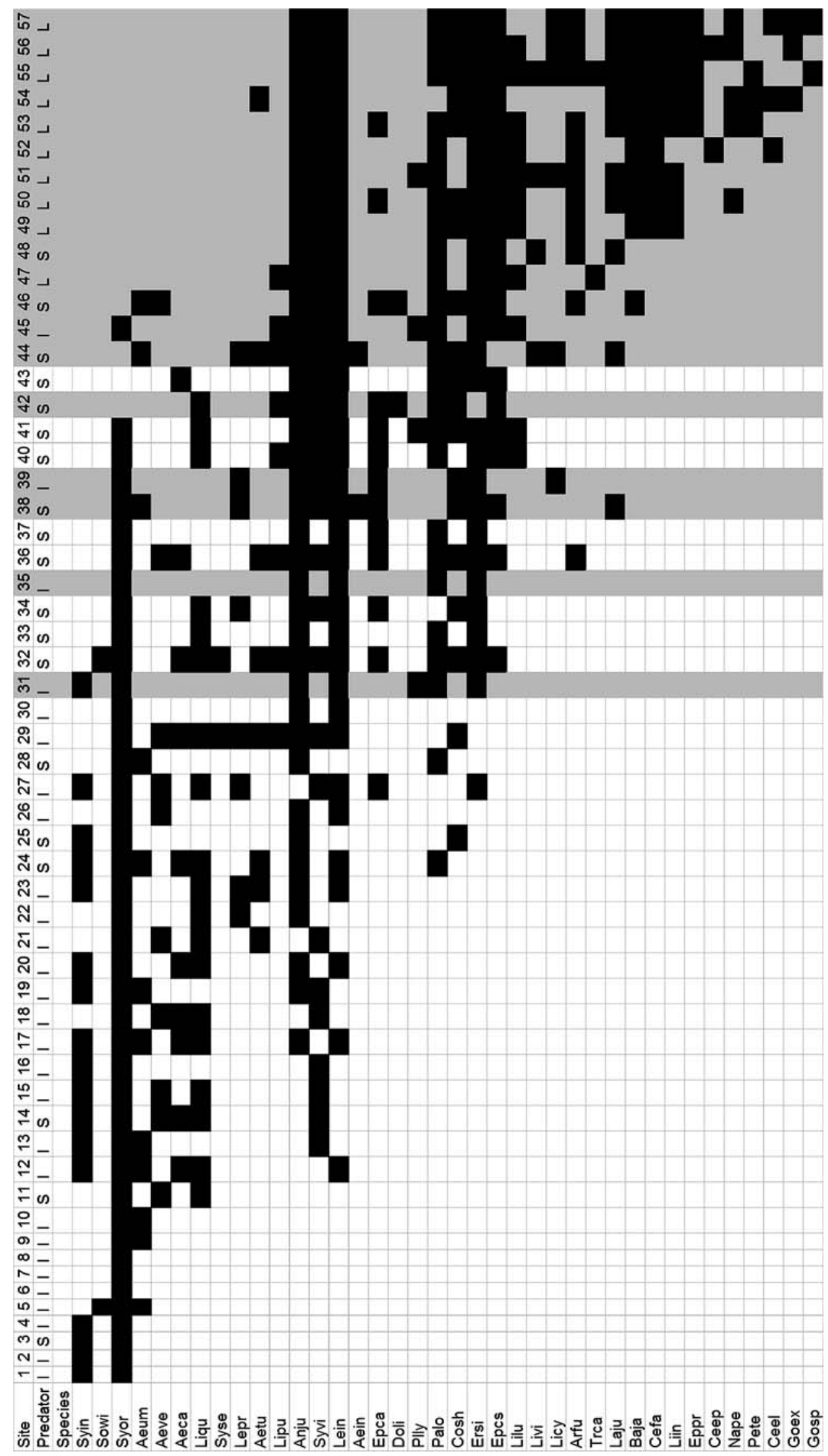


in nonpermanent sites on the ESGR (densities in pipe samples: $t=1.07, d f=20, P=0.298)$. In sites that dried every year of the survey, $49 \%$ of the mean richness in these sites (mean of 2.11 species) were species that cannot withstand pond drying. Therefore, a high proportion of the species interacting as larvae within these sites are derived from colonists from more permanent sites.

Across the six years of sampling 35 ponds on the ESGR, there were a total of 197 extinction events and 177 colonization events. Controlling for cumulative richness at a pond, there was no relationship between the habitat characteristics based on PC scores and the number of extinctions $(r=0.11$, $d f=32, P=0.54)$ or the number of colonizations $(r=-0.23$, $d f=32, \quad P=0.18)$. Extinction and colonization events occurred commonly and across the entire habitat gradient.

Only six of the 27 species collected on the ESGR were found in any one site for all six years surveyed. Consequently, only $22 \%$ of the regional diversity on the ESGR was maintained through strictly local recruitment, and these species are being maintained across different portions of the habitat gradient.

\section{Discussion}

Our results indicate that both niche- and dispersal-assembly processes affect the species distributions and richness patterns in this community. Richness of the larval dragonfly community was positively related to habitat area and permanence and negatively related to canopy cover. Species richness was higher in habitats with large-bodied fish than with either small-bodied fish or invertebrates when the effects of other measured environmental conditions are removed. The high degree of correlation between these variables (Table 2) makes it difficult to tease apart the contribution of each parameter to the overall richness patterns, but the picture which emerges is that within-habitat conditions are strong determinants of species richness. However, in semipermanent and temporary habitats, dispersal also had a strong effect on richness, suggesting that dispersal may be increasingly important as a mechanism affecting community structure as habitat permanence declines. This interpretation is supported by research in this system which has found that the habitat generalist species that are vulnerable to extinction from pond drying but which are frequently found in nonpermanent sites disperse more often and travel farther than species restricted to permanent lakes with large-bodied fish (McCauley 2007). Consequently, species richness in temporary and semipermanent habitats is best understood through a metacommunity perspective which provides a more complete understanding of the factors regulating community structure in this system than strictly local or dispersal-assembly perspectives.
Species distributions across the habitat gradient suggest that species sorting along the template of environmental conditions is a dominant pattern in this system. The coherence found in the ordinated matrix and the strong, positive correlation between habitat dissimilarity and community dissimilarity when controlling for distance both indicate species sorting across this gradient. The correlation between distance and community dissimilarity, controlling for habitat similarity, was also significant but relatively low in magnitude. Taken together, these results provide evidence that the observed species sorting was more strongly related to habitat conditions than to the spatial clumping of habitat types or a habitat by distance covariance.

The patterns of species sorting observed provide support for a niche-assembly model of community structure, and observed species distributions fit the predictions of the adaptive trade-offs model. Evidence that species sorting occurs in this system includes the finding of significant community boundary clumping in the ordinated matrix. Although ordination of the incidence matrix did not find significant species turnover, a prediction of the adaptive trade-offs model, there was community clumping with suites of species associated with alternative predator communities. These suites of species include nine that were found only in habitats with large-bodied fish and nine that were found only in habitats without large-bodied fish. This observation indicated a transition in community composition related to local predator conditions and provides support for predictions of the adaptive trade-offs model.

Further support for the adaptive trade-offs model comes from the finding that species that did not coexist with largebodied fish had shorter larval periods than those species which did co-occur with this predator type. Many of the species commonly restricted to habitats where small-bodied fish or invertebrates are top predators are species in the genera Sympetrum and Aeshna, which have an egg diapause stage that allows them to persist through drying but requires very rapid growth and development to reach maturity the next year. The absence of these species from a large number of sites where they could complete larval development but where they would encounter the most high-risk predators, large-bodied fish (McCauley 2007), may be driven by a trade-off between growth or development rates and predation risk mediated through activity level (Gotthard 2000; Werner and Anholt 1993; but see: McPeek 2004). A comparison of activity levels in nine species of libellulid dragonfly larvae found a negative association between activity level and use of large-bodied fish habitats (McCauley 2008). Johansson et al. (2006) found similar patterns in European lakes with and without fish, with actively foraging dragonfly species dominating in fishless lakes while less active species were more abundant in lakes with fish as the top predators. Similar trade-offs have been observed to 
structure species distributions in other systems, confining species with rapid growth and development to more disturbance-prone habitats with lower levels of predation risk (Johansson and Suhling 2004; Skelly 1995; Stoks and McPeek 2003; Wissinger et al. 1999; Woodward 1983). Adaptive trade-offs in vulnerability to different predator types may also restrict the distributions of species confined to habitats with large-bodied fish. However, tests of the mechanisms structuring these species' distributions indicate that predator vulnerabilities and dispersal limitation both act to limit the distributions of these species (McCauley 2007).

Patterns of species distributions did not support the developmental constraints model. We did not find nested species distributions in our analysis of the incidence matrix, which is one of the predictions of this model. Instead, many species utilized habitats which were consistent or intermittent sinks because hydroperiods were inadequate for meeting their larval developmental requirements, and species that were found in nonpermanent habitats did not have mean shorter larval periods than species that were confined to permanent sites. The presence of larvae in sites where they cannot complete development arises from the movement of adults into these sites from ponds in which they were able to complete development. Among the libellulid dragonflies, species with broad distributions across the habitat permanence and predator gradient have higher dispersal rates and move longer distances than species restricted to permanent lakes with large-bodied fish (McCauley 2007). These species are colonizing sites where they cannot complete development, in at least some periods, which is possibly due to risk spreading by the highly vagile adults, which have the potential to breed in multiple sites. Further evidence contradicting the developmental constraints hypothesis is that a number of species did not utilize those permanent sites where they could complete development but where they would also encounter predation pressure from large-bodied fish. Taken together, these data suggest a relatively poor fit between the species' larval developmental periods and their use of habitats that differ in their degree of permanence, and little support for the developmental constraints hypothesis.

One potential mechanism of species sorting we cannot address with our survey data is the extent to which patterns of species distributions are generated by habitat selection rather than species performance. The fit between developmental rate and habitat distribution across the predator transition parallels those found in other systems where species performance limits distributions, but this pattern could alternatively be generated or reinforced by habitat selection behavior. There is evidence that habitat selection related to canopy cover and predators can affect species distributions in other aquatic taxa (Resetarits et al. 2005). In dragonflies, the use of polarized light to locate ponds (Bernath et al. 2001, 2002) and evidence from colonization patterns in a field mesocosm study (McCauley 2005) suggest that habitat selection based on canopy cover may contribute to the observed decrease in species richness associated with this habitat variable by limiting the number of individuals that detect a closed canopy site. A similar mechanism might affect species distributions across predator communities. However, the limited data we currently have do not support this hypothesis. Observations of adults find that some species which are not found as larvae in lakes with largebodied fish nonetheless do breed at lakes with these fish (S.J. McCauley, personal observation). The experimental evidence that is currently available indicates that adult odonates cannot detect the presence of fish (McCauley 2005; McPeek 1989).

Species sorting was not, however, the only process affecting community structure in this system. Evidence for dispersal-assembly mechanisms, in particular mass effects, was found in the species composition of nonpermanent sites. Species that require water through at least one fall and winter of their larval development contributed a high proportion of the richness found in nonpermanent sites, including nearly half of the species collected from sites that dried every year and were consequently consistent population sinks. Three years of observations of the adult dragonflies breeding at sites off the ESGR indicated that many species repeatedly recolonize sites that rarely hold water long enough for them to complete development (McCauley, unpublished data). Dispersal between sites was also critical to maintaining regional diversity in this system. On the ESGR, fewer than a quarter of the species collected during this survey were maintained in the regional system by their consistent presence in one or more local sites. This pattern occurred across the habitat gradient. Even in permanent ponds, relatively few species were found to be consistently present in these sites. Although a solid body of theory indicates that dispersal is important to maintaining species diversity in local and regional systems (Tilman 1994; Lehman and Tilman 1997; Loreau and Mouquet 1999), empirical evidence for this has been limited (Vandvik and Goldberg 2006). Results from this system suggest that dispersal between fluctuating source and sink ponds are critical to maintaining diversity regionally, including in sites not subjected to regular disturbance.

We found a significant effect of distance on community compositional similarity, independent of habitat conditions, but our data provide little support for a neutral community model. The correlation between distance and community dissimilarity was positive and significant but considerably lower in magnitude $(r=0.08)$ than the distance-independent effects of habitat dissimilarity $(r=0.63)$. We are unaware of predictions about the relative magnitude of a 
distance effect predicted by neutral models, and it is possible that observations at a larger spatial scale might find a greater impact of distance. Nonetheless, habitat conditions clearly had a larger effect on community similarity than distance, and this, in conjunction with evidence of species sorting, does not support a neutral community hypothesis. Evidence from other work indicates that species that occur along different portions of the habitat permanence gradient differ in their adult dispersal behavior (McCauley 2007) and larval activity levels (McCauley 2008). Differences in both the habitat requirements of species and in the extent to which dispersal limits their distributions suggest that dispersal-assembly mechanisms are important in structuring this community but do not support a key component of neutral community models, functional equivalence of species. It may be that neutral community models are unlikely to fit when we examine communities across such a broad habitat gradient, making it plausible that although portions of a habitat gradient have functionally equivalent species, this is not the case across the full gradient occupied by this community.

Our results indicate that both species-sorting and dispersal-assembly mechanisms are critical to explaining patterns of richness and species distributions across habitat gradients. These results join a growing body of evidence that many natural systems are structured by processes occurring at both levels (e.g., Cottenie and De Meester 2004; Urban 2004; Ellis et al. 2006; Vanschoenwinkel et al. 2007; Driscoll 2008). Our results are also congruent with Cottenie's (2005) meta-analysis of 158 data sets on the extent to which communities are structured by environmental variables, spatial variables, or a combination of these variables. He found that most data sets $(44 \%)$ were structured by species sorting, while a sizeable fraction (29\%) were best explained by a combination of species sorting and one dispersal-assembly mechanism, mass effects. He also found that active dispersers are more affected by spatial factors than passive dispersers, while active dispersers from lake habitats were affected by a combination of spatial and environmental variables. The odonate system we studied falls in the range of mixed spatial and environmental effects expected based on Cottenie's (2005) synthesis.

Our study, however, is unique in examining how the importance of these processes may shift across a gradient of disturbance frequency, generated by pond drying, and predation risk. Given the generality of disturbance-biotic intensity gradients in both freshwater and terrestrial systems (Collins 2000; Connell 1978; Schneider and Frost 1996; Stoks and McPeek 2003; Tilman 1997; Wellborn et al. 1996; Wissinger et al. 1999), these results suggest some highly general conclusions about the conditions under which we expect niche- and dispersal-assembly processes to dominate. In permanent sites, which do not experience disturbance through drying but which have high-risk predators, species-sorting processes appear to be more critical than dispersal. In contrast, community structure in sites which experience more frequent disturbance from periodic drying were more affected by dispersal. Many species absent from permanent lakes possess traits that are likely to make them more vulnerable to the large-bodied fish in these sites, including high activity levels (McCauley 2008) and significantly shorter larval periods than species that do coexist with large-bodied fish. These strong associations between species' larval traits and their habitat distribution with respect to the top predator community supports a species-sorting hypothesis.

Surveying habitats with a broad range of hydroperiods and different predator communities necessitates sampling water bodies that differ widely in size. A critical assumption of our survey was that species detection probabilities were equivalent across habitats of varying sizes and that we were able to make robust estimates of the true diversity at different portions along this gradient. We adjusted our sampling efforts based on habitat size, but small ponds still receive relatively more intensive sampling in the surveys off the ESGR, and this was particularly true when comparing sampling intensities for the smallest ponds and the largest lakes. This may bias our results towards finding a greater fraction of the true richness in smaller ponds than in large lakes. Analysis of species accumulation curves and the fraction of singletons in ponds of different sizes suggest that our sample protocols resulted in similar detection probabilities for species across the range of habitat sizes surveyed, and that they were adequate to achieve good estimates of the richness of these habitats. The potential biases that can arise in combining survey data from a broad range of habitat types need to be considered in studies such as this one, and continual reassessments of sampling efficacy will improve our ability to interpret these results. However, surveys across broad gradients can also provide new insights that surveys of a smaller portion of the full gradient will not. For example, one result that emerges from our surveys, specific to freshwater systems but of great importance in these systems, is that the critical break in species distributions across the transition of top predator types was at the point of transition from small-bodied fish to large bodiedfish as top predators (Fig. 2). In this study region, most of the small-bodied fish habitats are dominated by a single species (eastern mudminnows), so whether this pattern is a species-specific effect or is generalizable to habitats with other small-bodied fish remains to be determined. This result, however, differs from the dominant paradigm of freshwater community structure across this gradient being principally influenced by the shift from conditions without fish to those with fish (e.g., Wellborn et al. 1996), and suggests that the influence of fish on the macroinvertebrate 
community may depend on the composition of the fish community. This pattern is revealed largely because of the breadth of our sampling across a habitat gradient.

Dispersal had a stronger effect on community structure at the less permanent end of the habitat gradient. Species richness in habitats which experience disturbance through drying events was strongly driven by the dispersal of individuals from more permanent sites into these habitats, suggesting that dispersal-assembly processes are more important in these sites than in permanent habitats. This result is unsurprising given the need for species to recolonize sites that experience drying. However, an unexpected result was the extent to which many species actually do recolonize intermittent sinks, and the extent to which species are not being maintained in a system by continual occupation of a few stable sites but by their occupation of multiple sites of varying stability. There were high levels of extinction and colonization across the gradient, although these estimates do not include the most temporally stable sites in the system (permanent lakes with large-bodied fish). We used liberal estimates of extinction and colonization events, and it is possible that some species at low densities were not detected, so we may have overestimated the number of extinction and colonization events. Nonetheless, the large numbers of such events and the known drying frequency of these habitats indicate that this is a highly dynamic community and one in which dispersal strongly affects community structure. Additionally, many sites were intermittent sources because of inter-annual variation in hydroperiod and shifts in the resident populations, suggesting that these dynamics may be critical to maintaining regional species richness.

The regular recolonization of sites that are at least intermittently sink habitats may be more common in dragonflies than in other taxa in these ponds, such as the amphibians, where there is considerable evidence that species-sorting mechanisms are important in structuring the community (Wellborn et al. 1996 and references therein; Werner et al. 2007). Consequently, even within the same habitats, taxa may be differentially affected by alternative mechanisms. These differences are likely to be related to the relative vagility of these taxa and the length of the adult stage. Unlike many species of amphibians, dragonfly adults in this region breed in only one season and cannot forgo breeding during years when pond drying is more likely. The relative vagility of dragonflies, their short adult life-spans, and the lack of suitable cues about the probability of habitat permanence available to them during their breeding periods, may make them more likely than amphibians to engage in riskspreading behavior by breeding at multiple sites that span the hydroperiod spectrum. The importance of the sourcesink dynamics that arise from this behavior suggest that changes in landscape connectivity could cause decreases in the species richness of this system, and highlights the value of survey data taken across broad habitat gradients. Data of this type are necessary to test theoretical models of community assembly and determine how multiple processes structure ecological communities.

Acknowledgments Many people provided useful comments on this manuscript at various stages in its development, including M. Benard, T. Brodin, J. Middlemas-Brown, D. Dritz, P. Epanchin, S. Fogarty, M. Fraker, D. Goldberg, B. Hazlett, M. Holyoak, S. Lawler, B. Luttbeg, T. Morgan, J. Saltz, S. Schneider, A. Sih, E. Silverman, W. White, and A. Zellmer. Too many researchers to name individually helped with sampling, and we are grateful to all of them. Access to the ESGR was provided by the Museum of Zoology and R. Nussbaum. J. Bolgos provided logistical support. This research was funded by NSF grants: DEB-9727014 to E. Werner, D. Skelly, R. Relyea, and K. Yurewicz, and DEB-0089809 to E. Werner and S. Peacor. S. McCauley received funding to sample water bodies off the ESGR from the Michigan Natural Heritage program funded by citizen contributions to the State of Michigan's Nongame Wildlife Fund (NH01-034), and from a Sigma Xi Grants-in-Aid of Research award. S. McCauley was supported by a University of Michigan Rackham Predoctoral Fellowship during the initial writing of this manuscript, and by a Center for Population Biology Postdoctoral Research Fellowship during the revision of this manuscript. This research complies with the current laws of the United States.

\section{References}

Bell G (2001) Ecology—neutral macroecology. Science 293:2413-2418

Bernath B, Szedenics G, Molnar G, Kriska G, Horvath G (2001) Visual ecological impact of "shiny black anthropogenic products" on aquatic insects: oil reservoirs and plastic sheets as polarized traps for insects associated with water. Arch Nat Conserv Landsc Res 40:89-109

Bernath B, Szedenics G, Wildermuth H, Horvath G (2002) How can dragonflies discern bright and dark waters from a distance? The degree of polarisation of reflected light as a possible cue for dragonfly habitat selection. Freshw Biol 47:1707-1719

Chase JM (2003) Community assembly: when should history matter? Oecologia 136:489-498

Collins SL (2000) Disturbance frequency and community stability in native tallgrass prairie. Am Nat 155:311-325

Colwell RK, Coddington JA (1994) Estimating terrestrial biodiversity through extrapolation. Philos Trans R Soc Lond B Biol Sci 345:101-118

Connell JH (1978) Diversity in tropical rain forests and coral reefshigh diversity of trees and corals is maintained only in a non-equilibrium state. Science 199:1302-1310

Cornell HV, Lawton JH (1992) Species interactions, local and regional processes, and limits to the richness of ecological communitiesa theoretical perspective. J Anim Ecol 61:1-12

Cottenie K (2005) Integrating environmental and spatial processes in ecological community dynamics. Ecol Lett 8:1175-1182

Cottenie K, De Meester L (2004) Metacommunity structure: synergy of biotic interactions as selective agents and dispersal as fuel. Ecology 85:114-119

Cottenie K, Nuytten N, Michels E, De Meester L (2001) Zooplankton community structure and environmental conditions in a set of interconnected ponds. Hydrobiologia 442:339-350

Driscoll DA (2008) The frequency of metapopulations, metacommunities and nestedness in a fragmented landscape. Oikos 117:297309 
Ellis AM, Lounibos LP, Holyoak M (2006) Evaluating the long-term metacommunity dynamics of tree hole mosquitoes. Ecology $87: 2582-2590$

Friedenberg NA (2003) Determinism in a transient assemblage: the roles of dispersal and local competition. Am Nat 162:586-596

Gilbert B, Lechowicz MJ (2004) Neutrality, niches, and dispersal in a temperate forest understory. Proc Natl Acad Sci USA 101:76517656

Gotelli NJ, Entsminger GL (2001) EcoSim: null models software for ecology, 7.0 edn. Acquired Intelligence Inc. \& Kesey-Bear, Jericho (see http://homepages.together.net/ gentsmin/ecosim.htm, last accessed 4 Sept 2008)

Gotthard K (2000) Increased risk of predation as a cost of high growth rate: an experimental test in a butterfly. J Anim Ecol 69:896-902

Holt RD (1997) On the evolutionary stability of sink populations. Evol Ecol 11:723-731

Holyoak M, Leibold MA, Holt RD (2005) Metacommunities: spatial dynamics and ecological communities. University of Chicago Press, Chicago

Hubbell SP (2001) The unified neutral theory of biodiversity and biogeography. Princeton University Press, Princeton

Jackson DA (1993) Stopping rules in principal components-analysisa comparison of heuristic and statistical approaches. Ecology 74:2204-2214

Johansson F, Suhling F (2004) Behaviour and growth of dragonfly larvae along a permanent to temporary water habitat gradient. Ecol Entomol 29:196-202

Johansson F, Englund G, Brodin T, Gardfjell H (2006) Species abundance models and patterns in dragonfly communities: effects of fish predators. Oikos 114:27-36

Kneitel JM, Miller TE (2003) Dispersal rates affect species composition in metacommunities of Sarracenia purpurea inquilines. Am Nat 162:165-171

Lehman CL, Tilman D (1997) Competition in spatial habitats. In: Tilman D, Kareiva P (eds) Spatial ecology: the role of space in population dynamics and interspecific interactions. Princeton University Press, Princeton, pp 185-203

Leibold MA, Mikkelson GM (2002) Coherence, species turnover, and boundary clumping: elements of meta-community structure. Oikos 97:237-250

Leibold MA et al (2004) The metacommunity concept: a framework for multi-scale community ecology. Ecol Lett 7:601-613

Loreau M, Mouquet N (1999) Immigration and the maintenance of local species diversity. Am Nat 154:427-440

McCauley SJ (2005) Species distributions in anisopteran odonates: effects of local and regional processes. Dissertation, University of Michigan, Ann Arbor

McCauley SJ (2007) The role of local and regional processes in structuring larval dragonfly distributions across habitat gradients. Oikos 116:121-133

McCauley SJ (2008) Slow, fast and in between: habitat distribution and behaviour of larvae in nine species of libellulid dragonfly. Freshw Biol 53:253-263

McPeek MA (1989) Differential dispersal tendencies among enallagma damselflies (odonata) inhabiting different habitats. Oikos 56:187-195

McPeek MA (2004) The growth/predation risk trade-off: So what is the mechanism? Am Nat 163:E88-E111

O'Brien M (2008) Michigan Odonata Survey (online): Odonata known from Michigan. http://insects.ummz.lsa.umich.edu/MICHODO/ michodolist.html, last accessed 4 Sept 2008

Patterson BD, Atmar W (1986) Nested subsets and the structure of insular mammalian faunas and archipelagoes. Biol J Linn Soc 28:65-82
Resetarits WJ Jr, Binckley CA, Chalcraft DR (2005) Habitat selection, species interactions, and processes of community assembly in complex landscapes: a metacommunity perspective. In: Holyoak M, Leibold MA, Holt RD (eds) Metacommunities spatial dynamics and ecological communities. The University of Chicago Press, Chicago, pp 374-398

Schneider D, Frost T (1996) Habitat duration and community structure in temporary ponds. J North Am Benthol Soc 15:64-86

Shmida A, Wilson MV (1985) Biological determinants of speciesdiversity. J Biogeogr 12:1-20

Shurin JB (2001) Interactive effects of predation and dispersal on zooplankton communities. Ecology 82:3404-3416

Skelly DK (1995) A behavioral trade-off and its consequences for the distribution of pseudacris treefrog larvae. Ecology 76:150-164

Skelly DK (1996) Pond drying, predators, and the distribution of Pseudacris tadpoles. Copeia 3:599-605

Skelly DK, Werner EE, Cortwright SA (1999) Long-term distributional dynamics of a Michigan amphibian assemblage. Ecology 80:2326-2337

Skelly DK, Bolden SR, Holland MP, Freidenburg LK, Freidenfelds NA, Malcolm TR (2006) Urbanization and disease in amphibians. In: S Collinge, C Ray (eds) Ecology of disease: community context and pathogen dynamics. Oxford University Press, Oxford

Stoks R, McPeek MA (2003) Predators and life histories shape Lestes damselfly assemblages along a freshwater habitat gradient. Ecology 84:1576-1587

Tilman D (1994) Competition and biodiversity in spatially structured habitats. Ecology 75:2-16

Tilman D (1997) Community invasibility, recruitment limitation, and grassland biodiversity. Ecology 78:81-92

Tofts R, Silvertown J (2002) Community assembly from the local species pool: an experimental study using congeneric species pairs. J Ecol 90:385-393

Urban MC (2004) Disturbance heterogeneity determines freshwater metacommunity structure. Ecology 85:2971-2978

Vandvik V, Goldberg DE (2006) Sources of diversity in a grassland metacommunity: quantifying the contribution of dispersal to species richness. Am Nat 168:157-167

Vanschoenwinkel B, De Vries C, Seaman M, Brendonck L (2007) The role of metacommunity processes in shaping invertebrate rock pool communities along a dispersal gradient. Oikos 116:12551266

Walker EM, Corbet PS (1978) The Odonata of Canada and Alaska. University of Toronto Press, Toronto

Wellborn GA, Skelly DK, Werner EE (1996) Mechanisms creating community structure across a freshwater habitat gradient. Annu Rev Ecol Syst 27:337-363

Werner EE, Anholt BR (1993) Ecological consequences of the tradeoff between growth and mortality-rates mediated by foraging activity. Am Nat 142:242-272

Werner EE, Skelly DK, Relyea RA, Yurewicz KL (2007) Amphibian species richness across environmental gradients. Oikos 116:1697-1712

Wissinger SA, Whiteman HH, Sparks GB, Rouse GL, Brown WS (1999) Foraging trade-offs along a predator-permanence gradient in subalpine wetlands. Ecology 80:2102-2116

Woodward BD (1983) Predator-prey interactions and breeding-pond use of temporary-pond species in a desert anuran community. Ecology 64:1549-1555

Wright JP, Flecker AS, Jones CG (2003) Local vs. landscape controls on plant species richness in beaver meadows. Ecology 84:31623173 